\title{
Long non-coding (lnc)RNA PAPAS overexpression inhibits tumor growth in papillary thyroid carcinoma by downregulating IncRNA HOTTIP
}

\author{
JUAN XIAO $^{1}$, ZHAOWEI BING $^{1}$, GE XIAO $^{2}$, YANG GUAN $^{3}$ and JIAN LUAN ${ }^{1}$ \\ ${ }^{1}$ Department of Endocrinology, Qingdao Municipal Hospital, Qingdao, Shandong 266000; \\ ${ }^{2}$ Department of Neurology, Qianfoshan Hospital Affiliated to Shandong University, Jinan, Shandong 250014; \\ ${ }^{3}$ Department of Static Distribution Center, Qingdao Municipal Hospital, Qingdao, Shandong 266000, P.R. China
}

Received February 13, 2019; Accepted August 13, 2019

DOI: $10.3892 / \mathrm{ol} .2020 .11323$

\begin{abstract}
The long non-coding (lnc)RNA pre-ribosomal RNA antisense transcripts (PAPAS) can repress ribosomal RNA synthesis, although its role in human disease is currently unknown. The present study aimed to investigate the function of PAPAS in papillary thyroid carcinoma (PTC). PAPAS was downregulated, while the lncRNA HOXA transcript at the distal tip (HOTTIP) was upregulated in patients with PTC. PAPAS and HOTTIP were inversely associated in PTC. PAPAS overexpression led to the downregulation of HOTTIP in PTC cells, while PAPAS expression was not significantly affected by HOTTIP overexpression, suggesting that PAPAS was upstream of HOTTIP. PAPAS expression level decreased, while HOTTIP expression level increased with the increase of clinical staging. PAPAS overexpression led to the inhibition of cell proliferation, while HOTTIP overexpression increased proliferation of PTC cells, and HOTTIP overexpression decreased the effects of PAPAS overexpression. IncRNA PAPAS overexpression may inhibit tumor growth in papillary thyroid carcinoma by downregulating lncRNA HOTTIP.
\end{abstract}

\section{Introduction}

Papillary thyroid carcinoma (PTC) is the most frequently diagnosed type of thyroid cancer, accounting for $>80 \%$ of all cases of thyroid cancer in 2010 worldwide (1). Thyroid cancer affects $\sim 4 \%$ of all patients with cancer in 2010 worldwide (2). However, the incidence rates of this disease have increased $>3$-fold over the past three decades (3). Therefore, thyroid cancer is predicted to be a major type of malignancy in the

Correspondence to: Dr Jian Luan, Department of Endocrinology, Qingdao Municipal Hospital, 1 Jiaozhou Road, Shibei, Qingdao, Shandong 266000, P.R. China

E-mail: jianluan201812@sohu.com

Key words: papillary thyroid carcinoma, long non-coding RNA pre-ribosomal RNA antisense transcripts, long non-coding RNA HOXA transcript at the distal tip, proliferation near future. PTC is usually treated with radioiodine ablation and surgery, and treatment outcomes are usually satisfactory (4). At present, $>95 \%$ of patients with PTC live $>5$ years after diagnosis (4). However, on occasion, certain aggressive forms of PTC can invade the lymph nodes, invade local or distant tissues, or even differentiate into lethal thyroid types of cancer (5), leading to poor survival rates.

Long non-coding RNAs (lncRNAs), ncRNAs of $>200$ nucleotides in length, form a group of functional non-protein coding RNA transcripts (6). IncRNAs were originally considered 'noise', although accumulating evidence over the past three decades has demonstrated that they are critical determinants in both physiological processes and pathological conditions, such as the occurrence and development of cancer (7-9). Therefore, regulation of lncRNA expression may provide new insights into the treatment or even prevention of cancer (10). However, the clinical application of lncRNAs is limited by the unknown function of the majority of lncRNAs. It has been previously reported that pre-ribosomal RNA antisense transcript (PAPAS) is able to repress ribosomal RNA (rRNA) synthesis (11). Our previous transcriptome analysis (12) revealed that PAPAS was downregulated in PTC tissues, and its expression levels were inversely associated with lncRNA HOXA transcript at the distal tip (HOTTIP), which is an oncogenic lncRNA in PTC (12). The present study assessed the involvement of PAPAS in PTC and investigated its association with HOTTIP.

\section{Materials and methods}

Patients. The present study enrolled 58 patients (21 males and 37 females) with PTC from the Qingdao Municipal Hospital (Qingdao, China), between January 2015 and January 2018. The mean age of the patients was $45.2 \pm 5.2$ years, (age range; 32-58 years). The inclusion criteria for the patients were as follows: i) Patients with PTC that had been diagnosed by imaging and histopathological examinations; ii) had no previous history of malignancy; iii) the patient was diagnosed with PTC, but had received no previous therapy; and iv) had normal thyroid function. The exclusion criteria for the patients were as follows: i) Patients had multiple clinical disorders 
diagnosed in addition to PTC; and ii) had received any form of treatment. Based on the American Joint Committee on Cancer system (13), there were $15,16,13$ and 14 cases at stage I, II, III and IV, respectively, in the present study. There were 26 cases at high grade and 32 cases at low grade (14). A total of 20 patients with thyroid goiter (8 males and 12 females; 34-56 years; $45.8 \pm 5.8$ years) were also selected during the same time period at the aforementioned hospital. All patients provided written informed consent prior to the start of the study. The present study was approved by the Ethics Committee of Qingdao Municipal Hospital.

Specimens and cell lines. Thyroid biopsy was performed on all patients in order to collect specimens of both tumor tissues and adjacent (within $2 \mathrm{~cm}$ from the tumor tissues) healthy tissues. All specimens were confirmed by three experienced pathologists at the aforementioned hospital (blinded). Thyroid biopsies were also obtained from the 20 patients with thyroid goiter. All thyroid biopsies from patients with thyroid goiter were stored in a liquid nitrogen sink at specimen library of the Qingdao Municipal Hospital.

IHH-4 and HTH-83 PTC cell lines and Nthy-ori 3-1 thyroid follicular epithelial cell line were used in the present study to perform all the in vitro cell experiments. Cells were bought from the American Type Culture Collection. DMEM supplemented with $100 \mathrm{mg} / \mathrm{ml}$ penicillin G, 10\% FBS (Sigma-Aldrich; Merck KGaA), and $100 \mathrm{U} / \mathrm{ml}$ streptomycin (Invitrogen; Thermo Fisher Scientific, Inc.) was used to culture cells in an incubator at $37^{\circ} \mathrm{C}$ with $5 \% \mathrm{CO}_{2}$ to reach about $80 \%$ confluence

Total RNA extraction and reverse transcription-quantitative $P C R$ (RT-qPCR). Total RNA in both tissue specimens and in vitro cells were extracted using TRIzol ${ }^{\circledR}$ reagent (Invitrogen; Thermo Fisher Scientific, Inc.) according to manufacturer's instructions. Tumor and adjacent healthy tissues were frozen in liquid nitrogen $\left(-196^{\circ} \mathrm{C}\right)$ prior to the addition of the TRIzol reagent. Following RNA extraction, SuperScript III Reverse Transcriptase kit (Thermo Fisher Scientific, Inc.) was used to perform the reverse transcription to synthesize cDNA through the following thermal conditions: $55^{\circ} \mathrm{C}$ for $30 \mathrm{~min}$ and $80^{\circ} \mathrm{C}$ for $10 \mathrm{~min}$. All PCR reaction mixtures were prepared using the SYBR Green Master mix (Bio-Rad Laboratories, Inc.). $18 \mathrm{~S}$ rRNA was used as the endogenous control. The $2^{-\Delta \Delta \mathrm{Cq}}$ method (15) was used to process the data. The primer sequences used were as follows: PAPAS forward, 5'-ATGGGGCCA AGATTGTGTCT-3' and reverse, 5'-AGACACAATCTTGGC CCCAT-3'; HOTTIP forward, 5'-AAGGCGGTTTTACATACT GGTC-3' and reverse, 5'-TAGCACCTGTAGTTGCCCATT CC-3'; 18S rRNA forward, 5'-GGCCCTGTAATTGGAATG AG-3' and reverse, 5'-CCAAGATCCAACTACGAGCTT-3'. The thermocycling conditions were as follows: $95^{\circ} \mathrm{C}$ for $5 \mathrm{~min}$, followed by 40 cycles of $95^{\circ} \mathrm{C}$ for $10 \mathrm{sec}$ and $55^{\circ} \mathrm{C}$ for $40 \mathrm{sec}$.

Vector constructions and cell transfection. pcDNA3.1 vectors expressing PAPAS and HOTTIP were constructed by Sangon Biotech Co., Ltd. An empty vector (pcDNA3.1) was used as negative control (NC). NC small interfering (si)RNA (5'-UUC UCCGAACGUGUCACGUUU-3') and HOTTIP siRNA (5'-GC CGCCGUGUCCACCGGCAGCU-3') were also obtained from
Sangon Biotech Co., Ltd. IHH-4 and HTH-83 PTC cells were harvested once they reached $70-80 \%$ confluence, and Lipofectamine 2000 (Thermo Fisher Scientific, Inc.) was used to transfect $10 \mathrm{nM}$ plasmid or $40 \mathrm{nM}$ siRNA into $10^{6}$ cells. The cells were harvested $24 \mathrm{~h}$ post-transfection prior to subsequent experiments.

Cell proliferation analysis. The effects of transfections on cell proliferation were analyzed using a Cell Counting Kit-8 (CCK-8; Sigma-Aldrich; Merck KGaA) assay according to manufacturer's instructions. Single cell suspensions were prepared using DMEM and cell concentration was diluted to $4 \times 10^{3}$ cells $/ \mathrm{ml}$. Cell culture was then performed using a 96-well plates $(100 \mu \mathrm{l}$ per well). The plates were incubated in an incubator at $37^{\circ} \mathrm{C}$ with $5 \% \mathrm{CO}_{2}$, followed by the addition of $10 \mu \mathrm{l}$ CCK- 8 solution every $24 \mathrm{~h}$ for $96 \mathrm{~h}$. Cells were then cultured for an additional $4 \mathrm{~h}$, followed by the addition of $10 \mu \mathrm{l}$ DMSO. Finally, optical density values at $450 \mathrm{~nm}$ were measured to analyze cell proliferation.

Statistical analysis. All statistical analysis was performed using GraphPad Prism 6 software (GraphPad Software, Inc.). Experiments were repeated in triplicate, and the results are presented as the mean \pm SD. Differences were assessed using one-way ANOVA followed by Tukey's test. Associations between the expression levels of two genes were analyzed by linear regression analysis. $\mathrm{P}<0.05$ was considered to indicate a statistically significant difference.

\section{Results}

Expression levels of PAPAS and HOTTIP are altered in tumor tissues of patients with PTC. Differential gene expression provides strong evidence for the involvement of certain genes in various pathological processes. Therefore, the present study investigated the expression of PAPAS and HOTTIP in both tumor and adjacent healthy tissues via RT-qPCR. Compared with the adjacent healthy tissues, expression levels of PAPAS were significantly lower in tumor tissues and thyroid biopsies from patients with thyroid goiter $(\mathrm{P}<0.05$; Fig. 1A). By contrast, lncRNA HOTTIP levels were upregulated in tumor tissues and thyroid biopsies form thyroid goiter patients than in adjacent healthy tissues $(\mathrm{P}<0.05$; Fig. 1B). However, no significant differences were observed between tumor tissues and thyroid biopsies from patients with thyroid goiter.

PAPAS and HOTTIP are inversely associated in PTC. Correlations between PAPAS and HOTTIP were analyzed by linear regression analysis. It was observed that PAPAS and HOTTIP were inversely and significantly associated in tumor tissues $(\mathrm{P}<0.0001$; Fig. 2A). However, the expression levels of PAPAS and HOTTIP in adjacent healthy tissues were not significantly associated ( $\mathrm{P}=0.1420$; Fig. $2 \mathrm{~B})$, indicating that PAPAS and HOTTIP were associated specifically under pathological conditions.

PAPAS is an upstream negative regulator of HOTTIP in PTC cell lines. Expression levels of PAPAS and HOTTIP in IHH-4, HTH-83 and Nthy-ori 3-1 cell lines were measured by RT-qPCR. Compared with Nthy-ori 3-1 cells, PAPAS was 

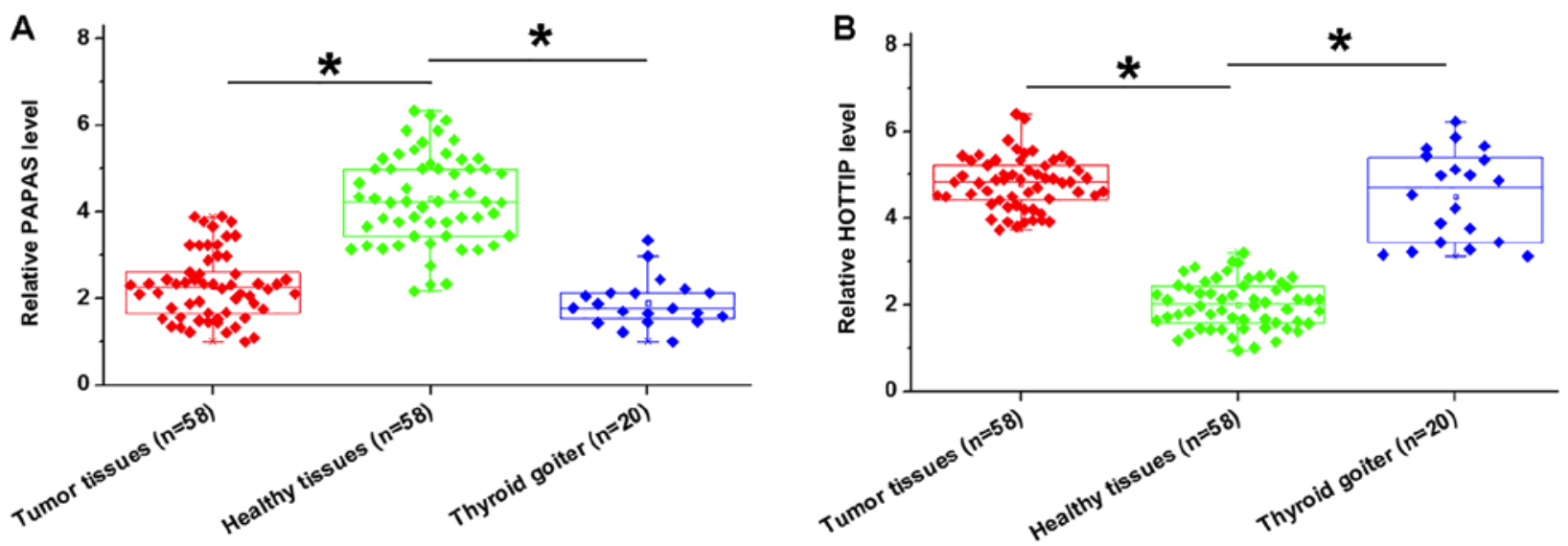

Figure 1. Expression levels of PAPAS and HOTTIP are altered in the tumor tissues of patients with PTC. Reverse transcription-quantitative PCR revealed that (A) PAPAS was downregulated, while (B) long non-coding RNA HOTTIP was upregulated in tumor tissues and thyroid biopsies from patients with thyroid goiter compared with adjacent healthy tissues of patients with papillary thyroid carcinoma. No significant differences were observed between thyroid biopsies from patients with thyroid goiter and thyroid biopsies from patients with PTC. ${ }^{*} \mathrm{P}<0.05$ vs. healthy tissues. PAPAS, pre-ribosomal RNA antisense transcripts; HOTTIP, HOXA transcript at the distal tip; PTC, papillary thyroid carcinoma.
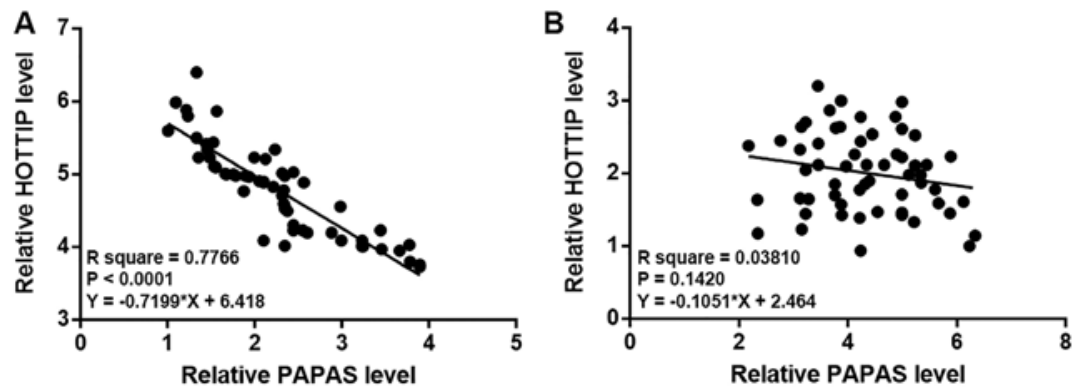

Figure 2. PAPAS and HOTTIP are inversely associated in papillary thyroid carcinoma. The linear regression analysis revealed that PAPAS and HOTTIP were inversely associated in (A) tumor tissues, but (B) not in adjacent healthy tissues. PAPAS, pre-ribosomal RNA antisense transcripts; HOTTIP, HOXA transcript at the distal tip.

downregulated $(\mathrm{P}<0.05$; Fig. S1A) and HOTTIP was upregulated $(\mathrm{P}<0.055$; Fig. S1B) in IHH-4 and HTH-83 cells. In order to further investigate the interactions between PAPAS and HOTTIP, PAPAS and HOTTIP vectors were transfected into the IHH-4 and HTH- 83 cell lines. The RT-qPCR results revealed that PAPAS and HOTTIP were overexpressed at $24 \mathrm{~h}$ after transfection in the IHH- 4 and HTH- 83 cell lines $(\mathrm{P}<0.05$; Fig. 3A). Compared with the untransfected (C) and NC groups, cells overexpressing PAPAS exhibited significantly downregulated expression levels of HOTTIP $(\mathrm{P}<0.05$; Fig. 3B). However, the expression level of PAPAS was not significantly altered in cells overexpressing HOTTIP (Fig. 3C). HOTTIP siRNA silencing was achieved in both IHH-4 and HTH-83 cells ( $\mathrm{P}<0.05$; Fig. $\mathrm{S} 2 \mathrm{~A})$. Compared with the $\mathrm{C}$ and $\mathrm{NC}$ groups, HOTTIP siRNA silencing failed to significantly affect the expression of PAPAS in these cells (Fig. S2B).

Expression levels of PAPAS and HOTTIP are affected by clinical stage. Comparison among patients with different clinical stages revealed that expression levels of PAPAS decreased significantly along with the increase in clinical staging $(\mathrm{P}<0.05 ;$ Fig. 4A). In contrast, HOTTIP expression level increased significantly with the increase of clinical staging $(\mathrm{P}<0.05$; Fig. 4B). The present results indicated that PAPAS and HOTTIP are involved in the progression of PTC.
PAPAS regulates PTC cell proliferation through HOTTIP. Compared with $\mathrm{C}$ and $\mathrm{NC}$ groups, PAPAS overexpression led to decreased cell proliferation, whereas HOTTIP overexpression increased the proliferation of PTC cells, and HOTTIP overexpression attenuated the effects of PAPAS overexpression in both IHH-4 and HTH-83 cells ( $\mathrm{P}<0.05$; Fig. 5).

\section{Discussion}

Although PAPAS has been reported to play a critical role in rRNA synthesis (11), its role in human disease remains unknown. The present study revealed that PAPAS was downregulated in PTC tissues and PTC cell lines, and played a tumor suppressive role in this disease. However, HOTTIP was upregulated in both PTC tissues and PTC cell lines. In addition, the role of PAPAS in PTC was found to be mediated by the downregulation of oncogenic HOTTIP.

Accumulating evidence demonstrated that HOTTIP promotes the development and progression of different types of cancer, such as pancreatic cancer and esophageal cancer $(16,17)$. HOTTIP is involved in cancer development and progression primarily by regulating cancer cell behaviors via its interactions with downstream tumor suppression of oncogenic pathways, such as HOXA13 and the epithelial-to-mesenchymal transition pathways $(16,17)$. In a recent study, Yuan et al (12) 

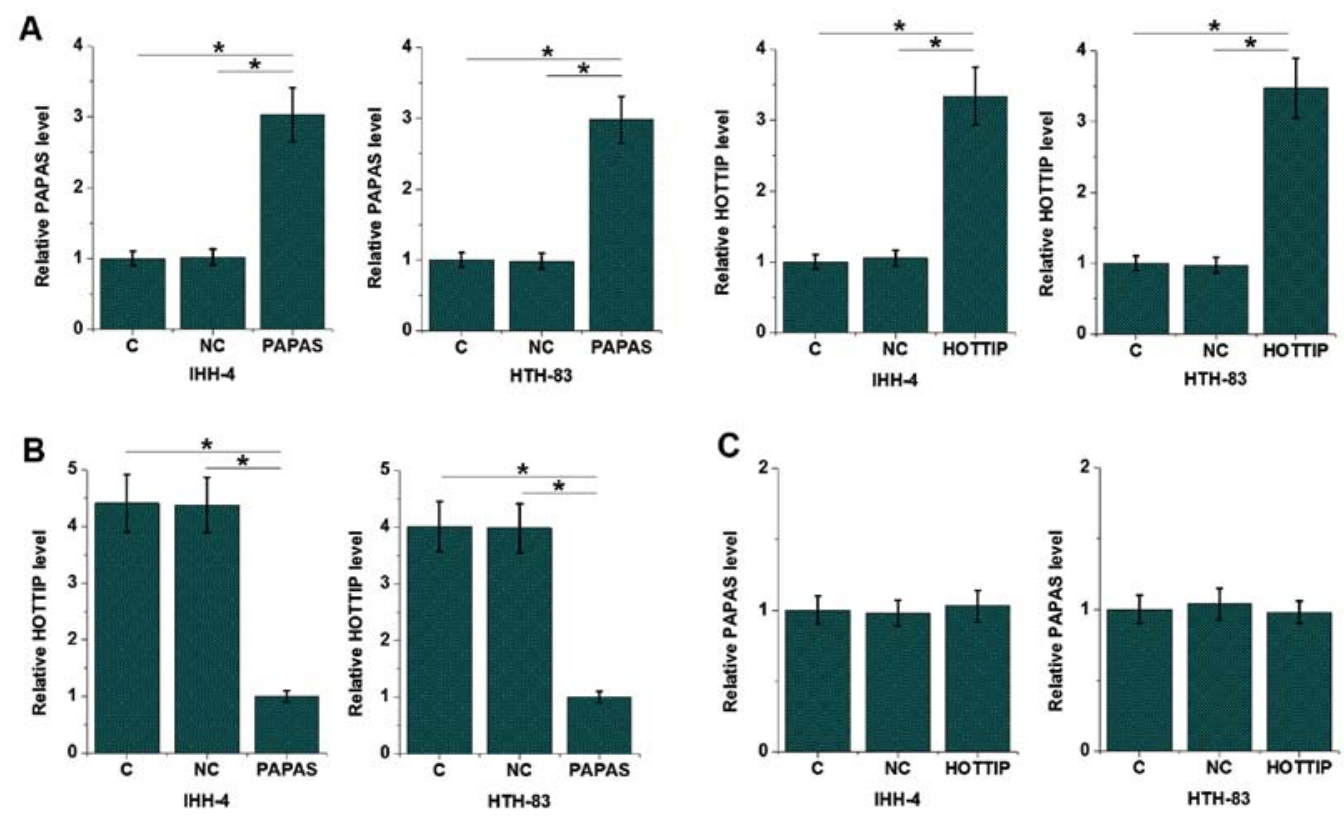

Figure 3. PAPAS is an upstream negative regulator of HOTTIP in IHH-4 and HTH-83 cell lines. (A) PAPAS and HOTTIP were upregulated $24 \mathrm{~h}$ after transfection. Compared with the $\mathrm{C}$ and NC groups, (B) PAPAS overexpression led to the downregulation of HOTTIP in IHH-4 and HTH-83 cells, while (C) HOTTIP overexpression failed to significantly affect PAPAS expression. ${ }^{*} \mathrm{P}<0.05$. PAPAS, pre-ribosomal RNA antisense transcripts; HOTTIP, HOXA transcript at the distal tip; C, untransfected control; NC, negative control.
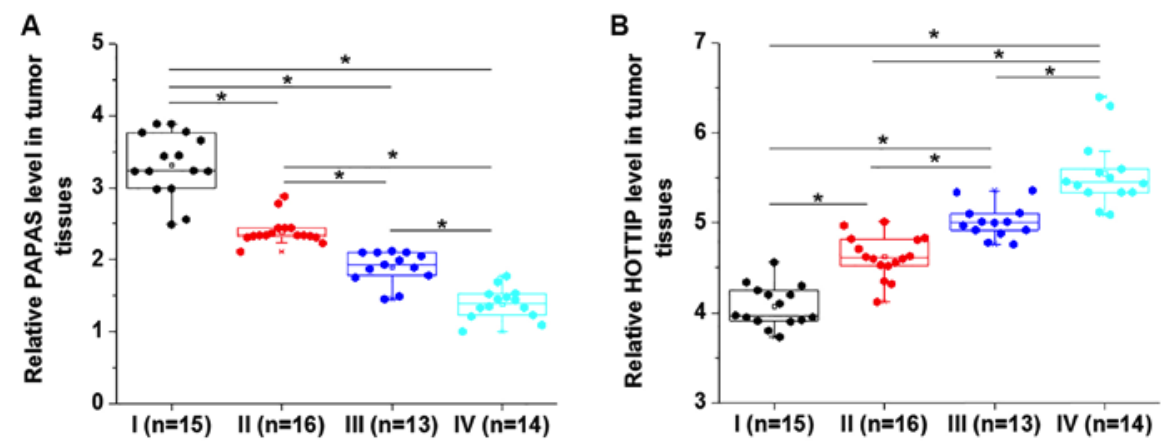

Figure 4. Expression levels of PAPAS and HOTTIP are affected by clinical stage. (A) PAPAS expression level decreased, while (B) HOTTIP expression level increased with the increase of clinical staging. ${ }^{~} \mathrm{P}<0.05$. PAPAS, pre-ribosomal RNA antisense transcripts; HOTTIP, HOXA transcript at the distal tip.
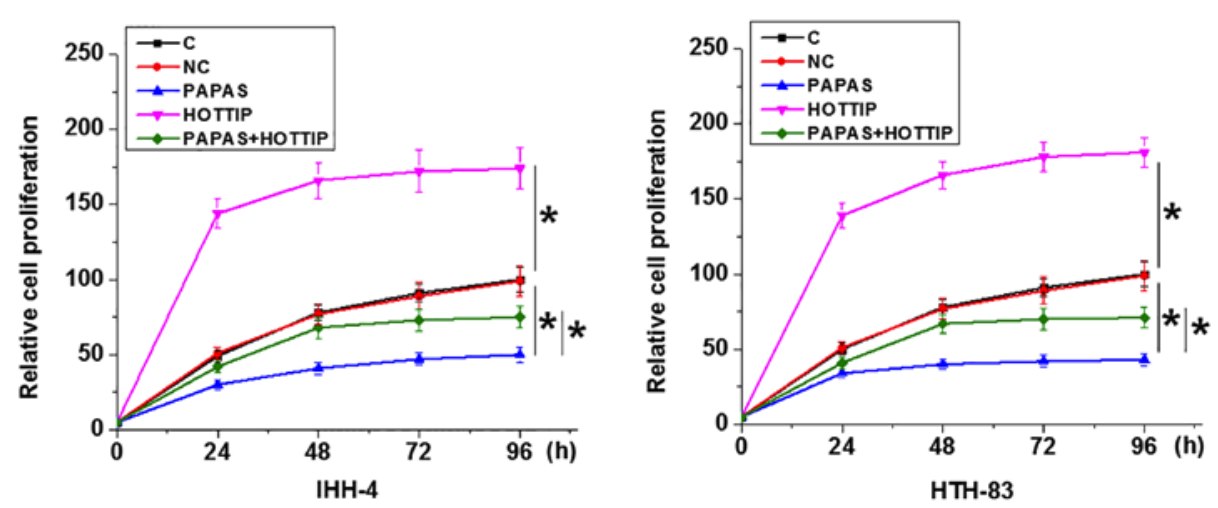

Figure 5. PAPAS regulates IHH-4 and HTH-83 cell proliferation via HOTTIP. The in vitro cell proliferation assay data revealed that PAPAS overexpression led to decreased cell proliferation, while HOTTIP overexpression led to increased proliferation of papillary thyroid carcinoma cells, and HOTTIP overexpression attenuated the effects of PAPAS overexpression. ${ }^{*} \mathrm{P}<0.05$. PAPAS, pre-ribosomal RNA antisense transcripts; HOTTIP, HOXA transcript at the distal tip; C, control; $\mathrm{NC}$, negative control.

reported that HOTTIP was upregulated in PTC and was able to promote cancer cell proliferation, invasion and migration through the downregulation of microRNA-637, which inhibits cancer development (12). Consistent with previous results (12), 
the present study observed the upregulated HOTTIP in PTC tissues, and the overexpression of HOTTIP led to significantly increased cancer cell proliferation. Therefore, the present study further confirmed the oncogenic role of HOTTIP in PTC.

To the best of our knowledge, the present study is the first to report the tumor suppressive role of PAPAS in PTC. IncRNAs participate in cancer biology, primarily by interacting with downstream oncogenes or tumor suppressors $(18,19)$. Notably, the present study indicated that PAPAS could serve a role in cancer development acting as an upstream inhibitor of HOTTIP in PTC. However, the interaction between PAPAS and HOTTIP was found to be likely indirect, due to the lack of association between the expression levels of these two lncRNAs in tumor adjacent healthy tissues. HOTTIP overexpression only partially attenuated the inhibitory effects of PAPAS overexpression on cancer cell proliferation. Therefore, it is likely that PAPAS may interact with multiple downstream effectors to achieve a fine regulation of PTC cell behaviors.

However, the present study is limited by the small sample size. In addition, studies with in vivo animal models are required in order to further validate the conclusions from the present study. However, the present study provided evidence of the existence of the interactions between different lncRNAs in cancer biology. The present results provide new insights into cancer biology. However, the molecular mechanisms underlying the interactions between lncRNAs are unclear.

PAPAS and HOTTIP were also dysregulated in thyroid goiter. Therefore, the interaction between PAPAS and HOTTIP is unlikely to be cancer-specific. Future studies should aim to investigate the involvement of PAPAS and HOTTIP in other types of thyroid disease.

In conclusion, PAPAS was downregulated in PTC and may inhibit PTC cell proliferation by downregulating HOTTIP.

\section{Acknowledgements}

Not applicable.

\section{Funding}

No funding was received.

\section{Availability of data and materials}

The datasets used and/or analyzed during the current study are available from the corresponding author on reasonable request.

\section{Authors' contributions}

JX and JL designed the experiments. JX and ZB performed experiments. GX and YG analyzed the data. JL drafted the manuscript. All authors approved the manuscript.

\section{Ethics approval and consent to participate}

The present study was approved by Ethics Committee of Qingdao Municipal Hospital (Qingdao, China). All patients provided written informed consent prior to the study start.

\section{Patient consent for publication}

Not applicable.

\section{Competing interests}

The authors declare that they have no competing interests.

\section{References}

1. Lloyd RV, Buehler D and Khanafshar E: Papillary thyroid carcinoma variants. Head Neck Pathol 5: 51-56, 2011.

2. LiVolsi VA: Papillary thyroid carcinoma An update. Mod Pathol 24 (Suppl 2): S1-S9, 2011.

3. Cancer Genome Atlas Research NetworkIntegrated genomic characterization of papillary thyroid carcinoma. Cell 159: 676-690, 2014.

4. Morris LG, Shaha AR, Tuttle RM, Sikora AG and Ganly I: Tall-cell variant of papillary thyroid carcinoma: A matched-pair analysis of survival. Thyroid 20: 153-158, 2010.

5. Jendrzejewski J, Thomas A, Liyanarachchi S, Eiterman A, Tomsic J, He H, Radomska HS, Li W, Nagy R, Sworczak K and de la Chapelle A: PTCSC3 is involved in papillary thyroid carcinoma development by modulating S100A4 gene expression. J Clin Endocrinol Metab 100: E1370-E1377, 2015.

6. Fatica A and Bozzoni I: Long non-coding RNAs New players in cell differentiation and development. Nat Rev Genet 15: 7-21, 2014.

7. Spizzo R, Almeida MI, Colombatti A and Calin GA: Long non-coding RNAs and cancer A new frontier of translational research. Oncogene 31: 4577-4587, 2012.

8. Gutschner T and Diederichs S: The hallmarks of cancer A long non-coding RNA point of view. RNA Biol 9: 703-719, 2012.

9. Shi X, Sun M, Liu H, Yao Y and Song Y: Long non-coding RNAs A new frontier in the study of human diseases. Cancer Lett 339: 159-166, 2013.

10. Qi P and Du X: The long non-coding RNAs, a new cancer diagnostic and therapeutic gold mine. Mod Pathol 26: 155-165, 2013.

11. Zhao Z, Sentürk N, Song C and Grummt I: lncRNA PAPAS tethered to the rDNA enhancer recruits hypophosphorylated CHD4/NuRD to repress rRNA synthesis at elevated temperatures. Genes Dev 32: 836-848, 2018.

12. Yuan Q, Liu Y, Fan Y, Liu Z, Wang X, Jia M, Geng Z, Zhang J and $\mathrm{Lu} \mathrm{X}$ : LncRNA HOTTIP promotes papillary thyroid carcinoma cell proliferation, invasion and migration by regulating miR- 637 . Int J Biochem Cell Biol 98: 1-9, 2018.

13. Kim TH Kim YN, Kim HI, Park SY, Choe JH, Kim JH, Kim JS, Oh YL, Hahn SY, Shin JH, et al: Prognostic value of the eighth edition AJCC TNM classification for differentiated thyroid carcinoma. Oral Oncol 71: 81-86, 2017.

14. Hakala T and Kholová I: Changes in classification of follicular thyroid cancers. Thyroid 26: 866, 2016.

15. Livak KJ and Schmittgen TD: Analysis of relative gene expression data using real-time quantitative PCR and the 2(-Delta Delta C(T)) method. Methods 25: 402-408, 2001.

16. Li Z, Zhao X, Zhou Y, Liu Y, Zhou Q, Ye H, Wang Y, Zeng J, Song Y, Gao W, et al: The long non-coding RNA HOTTIP promotes progression and gemcitabine resistance by regulating HOXA13 in pancreatic cancer. J Transl Med 13: 84, 2015.

17. Chen X, Han H, Li Y, Zhang Q, Mo K and Chen S: Upregulation of long noncoding RNA HOTTIP promotes metastasis of esophageal squamous cell carcinoma via induction of EMT. Oncotarget 7: 84480-84485, 2016.

18. Schmitt AM and Chang HY: Long noncoding RNAs in cancer pathways. Cancer Cell 29: 452-463, 2016.

19. Huarte M: The emerging role of lncRNAs in cancer. Nat Med 21: 1253-1261, 2015.

This work is licensed under a Creative Commons Attribution-NonCommercial-NoDerivatives 4.0 International (CC BY-NC-ND 4.0) License. 